Urologe 2020 · 59:607-612

https://doi.org/10.1007/s00120-020-01212-x

Online publiziert: 27. April 2020

(c) Springer Medizin Verlag GmbH, ein Teil von Springer Nature 2020

Im Mittelpunkt der öffentlichen Wahrnehmung steht derzeit das neuartige, erstmals im Dezember 2019 in Wuhan/ Volksrepublik China aufgetretene Coronavirus SARS-CoV-2 (2019-nCoV) und die durch das Virus ausgelöste Atemwegserkrankung COVID-19. ${ }^{1}$

Zudem traten am 1. März 2020 die durch das Gesetz für den Schutz vor Masern und zur Stärkung der Impfprävention (Masernschutzgesetz) ${ }^{2}$ eingeführten Änderungen des Infektionsschutzgesetzes in Kraft. Hintergrund der Änderungen sind die in den letzten Jahren deutlich gestiegenen Masernfallzahlen, wobei Masern nicht nur zu den ansteckendsten Infektionskrankheiten des Menschen zählen, sondern - anders als verbreitet angenommen - keine harmlose Krankheit darstellen, sondern schwer verlaufen und Komplikationen und Folgeerkrankungen nach sich ziehen können. ${ }^{3}$ Der Gesetzgeber sah sich daher veranlasst, zum Schutz der öffentlichen Gesundheit Regelungen zu implementieren, wonach Personen in bestimmten Einrichtungen entweder einen ausreichenden Impfschutz gegen Masern oder aber

\footnotetext{
1 Das Gesetz zum Schutz der Bevölkerung bei einer epidemischen Lage von nationaler Tragweite vom 27.03.2020 konnte im Rahmen der Korrekturfahnen noch berücksichtigt werden.

2 Gesetz für den Schutz vor Masern und zur Stärkung der Impfprävention vom 20.02.2020, BGBI. I2020, 148.

3 So der Gesetzesentwurf der Bundesregierung für den Schutz vor Masern und zur Stärkung der Impfprävention, BT-Drucks. 19/13452, 1.
}

Dieser Beitrag wurde zuerst publiziert in Notfall Rettungsmed 2020 https://doi.org/10.1007/ s10049-020-00698-4.

P. M. Lissel

München, Deutschland

\title{
Infektionsschutzrecht nach Inkrafttreten des Masernschutzgesetzes
}

eine Immunität gegen Masern aufweisen müssen.

Der nachfolgende Beitrag stellt die aktuelle Rechtslage, insbesondere das seit dem letzten Überblicksbeitrag ${ }^{4}$ wiederholt novellierte Infektionsschutzgesetz $(\text { IfSG })^{5}$ unter besonderer Berücksichtigung der Vorschriften des Masernschutzgesetzes - hierbei bezogen auf Gesundheitseinrichtungen - dar.

\section{Einführung}

Zweck des Infektionsschutzrechts ist es, übertragbaren Krankheiten beim Menschen vorzubeugen, Infektionen frühzeitig zu erkennen und ihre Weiterverbreitung $\mathrm{zu}$ verhindern. ${ }^{6}$ Zur Erreichung dieser Ziele arbeiten zahlreiche Stellen, insbesondere die Behörden des Bundes, der Länder und der Kommunen sowie Ärzte, Tierärzte, Krankenhäuser und wissenschaftliche Einrichtungen, zusammen ( $\$ 1$ IfSG).

Besondere Bedeutung kommt dabei dem Robert Koch-Institut (RKI) zu. Das zum Geschäftsbereich des Bundesministeriums für Gesundheit gehörende Bundesinstitut ist als selbständige Bundesoberbehörde die nationale Behörde zur Vorbeugung übertragbarer Krankheiten sowie zur frühzeitigen Erkennung und

\footnotetext{
${ }^{4}$ Lissel, Infektionsschutzrecht, Notfall Rettungsmed 2018, 139-142= Der Pneumologe, 2018, 354-357.

5 BGBI. I 2000, 1045, zuletzt geändert durch Gesetz vom 27.03.2020, BGBI.I 2020,587.

${ }^{6}$ Vgl. ausführlich Lissel, Infektionsschutzrecht, in: Rieger et al., Heidelberger Kommentar Arztrecht Krankenhausrecht Medizinrecht (HKAKM), 72. Aktualisierung 2018,Ziffer 2605.
}

Verhinderung der Weiterverbreitung von Infektionen. Es arbeitet mit den jeweils zuständigen Bundesbehörden, den zuständigen Landesbehörden, den nationalen Referenzzentren, weiteren wissenschaftlichen Einrichtungen und Fachgesellschaften zusammen. Auf Ersuchen einer obersten Landesgesundheitsbehörde kann das RKI den zuständigen Stellen bei Maßnahmen zur Überwachung, Verhütung und Bekämpfung von bedrohlichen übertragbaren Krankheiten Amtshilfe leisten (\$4 IfSG). Im Fall einer epidemischen Lage von nationaler Tragweite koordiniert das RKI die Zusammenarbeit zwischen den Ländern und zwischen den Ländern und dem Bund sowie weiteren beteiligten Behörden und Stellen und tauscht Informationen aus (\$ 5 IfSG).

\section{Begriffsbestimmungen}

Gemäß den Begriffsdefinitionen des $₫ 2$ IfSG ist eine übertragbare Krankheit eine durch Krankheitserreger oder deren toxische Produkte, die unmittelbar oder mittelbar auf den Menschen übertragen werden, verursachte Krankheit. Eine bedrohliche übertragbare Krankheit ist eine übertragbare Krankheit, die aufgrund klinisch schwerer Verlaufsformen oder ihrer Ausbreitungsweise eine schwerwiegende Gefahr für die Allgemeinheit verursachen kann.

Eine Person, die an einer übertragbaren Krankheit erkrankt ist, wird als Kranker, eine Person, bei der Symptome bestehen, die das Vorliegen einer übertragbaren Krankheit vermuten lassen, wird als Krankheitsverdächtiger bezeichnet. Ausscheider ist eine Person, die Krankheitserreger ausscheidet und 
damit eine Ansteckungsquelle für die Allgemeinheit sein kann, ohne krank oder krankheitsverdächtig zu sein. Als Ansteckungsverdächtiger wird eine Person bezeichnet, von der anzunehmen ist, dass sie Krankheitserreger aufgenommen hat, ohne krank, krankheitsverdächtig oder Ausscheider zu sein. Hierfür ist Voraussetzung, dass die Vermutung, eine Person habe Krankheitserreger aufgenommen, wahrscheinlicher ist als das Gegenteil.?

\section{Meldepflichten und zur Meldung verpflichtete Personen 8}

Namentlich meldepflichtig sind gemäß $\$ 6$ Abs. 1 Satz 1 IfSG:

1. der Verdacht einer Erkrankung, die Erkrankung sowie der Tod in Bezug auf die folgenden Krankheiten:

a) Botulismus,

b) Cholera,

c) Diphtherie,

d)humane spongiforme Enzephalopathie, außer familiär-hereditärer Formen,

e) akute Virushepatitis,

f) enteropathisches hämolytischurämisches Syndrom (HUS),

g) virusbedingtes hämorrhagisches Fieber,

h)Keuchhusten,

i) Masern,

j) Meningokokkenmeningitis oder -sepsis,

k) Milzbrand,

l) Mumps,

mPest

n)Poliomyelitis,

o) Röteln einschließlich Rötelnembryopathie,

p)Tollwut,

q) Typhus abdominalis oder Paratyphus,

r) Windpocken,

s) zoonotische Influenza,

1a die Erkrankung und der Tod in Bezug auf folgende Krankheiten:

\footnotetext{
7 BVerwG, NJW 2012, 2823.

${ }^{8}$ Weitere Meldepflichten ergeben sich aus $\S 7$ IfSG (meldepflichtige Nachweise von zahlreichen Krankheitserregern), auf diese wird in weiterer Folge nicht näher eingegangen.
}

a) behandlungsbedürftige Tuberkulose, auch wenn ein bakteriologischer Nachweis nicht vorliegt,

b)Clostridioides-difficile-Infektion mit klinisch schwerem Verlauf, wobei dieser noch näher definiert wird,

2. der Verdacht auf und die Erkrankung an einer mikrobiell bedingten Lebensmittelvergiftung oder an einer akuten infektiösen Gastroenteritis, wenn

a) eine Person betroffen ist, die bestimmte, im Einzelnen genannte Lebensmittel herstellt, behandelt oder in Verkehr bringt, wenn sie dabei mit diesen in Berührung kommt oder in Küchen von Gaststätten und sonstigen Einrichtungen mit oder zur Gemeinschaftsverpflegung tätig ist,

b)zwei oder mehr gleichartige Erkrankungen auftreten, bei denen ein epidemischer Zusammenhang wahrscheinlich ist oder vermutet wird,

3. der Verdacht einer über das übliche Ausmaß einer Impfreaktion hinausgehenden gesundheitlichen Schädigung,

4. die Verletzung eines Menschen durch ein tollwutkrankes, -verdächtiges oder -ansteckungsverdächtiges Tier sowie die Berührung eines solchen Tieres oder Tierkörpers,

5. das Auftreten einer bedrohlichen übertragbaren Krankheit, die nicht bereits nach den Nummern 1 bis 4 meldepflichtig ist.

Nach $₫ 6$ Abs. 2 IfSG ist über die Meldepflicht bei Masern ( $\$ 6$ Abs. 1 Satz 1 Nr. 1 Buchst. i IfSG) hinaus zu melden, wenn Personen an einer subakuten sklerosierenden Panenzephalitis infolge einer Maserninfektion erkranken oder versterben. Ferner ist über die Meldung nach $\$ 6$ Abs. 1 Satz 1 Nr. 1a Buchst. a IfSG hinaus zu melden, wenn Personen, die an einer behandlungsbedürftigen Lungentuberkulose erkrankt sind, eine Behandlung verweigern oder abbrechen.
Nichtnamentlich zu melden ist das Auftreten von zwei oder mehr nosokomialen Infektionen, bei denen ein epidemischer Zusammenhang wahrscheinlich ist oder vermutet wird ( $\$ 6 \mathrm{Abs}$. 3 IfSG).

Gemäß $₫ 15$ IfSG kann das Bundesministerium für Gesundheit die Meldepflicht durch Rechtsverordnung aufheben, einschränken oder erweitern oder auf andere übertragbare Krankheiten ausdehnen, soweit die epidemische Lage dies zulässt oder erfordert. Aufgrund dieser Ermächtigungsnorm wurde mit Wirkung zum 1. Februar 2020 die Meldepflicht ausgedehnt auf den Verdacht einer Erkrankung, die Erkrankung sowie den Tod in Bezug auf eine Infektion, die durch das erstmals im Dezember 2019 in Wuhan/Volksrepublik China aufgetretene neuartige Coronavirus (2019-nCoV) hervorgerufen wird. ${ }^{9}$ Hingegen wurde die bislang bestehende Verordnung zur Anpassung der Meldepflichten an die epidemische Lage ${ }^{10}$ mit Wirkung zum 1. März 2020 aufgehoben, ${ }^{11}$ die dortigen Meldepflichten wurden in das IfSG überführt.

Durch $₫ 15$ IfSG werden auch die einzelnen Bundesländer ermächtigt, die Meldepflicht durch Rechtsverordnung zu erweitern oder auf andere übertragbare Krankheiten auszudehnen, sofern die epidemische Lage dies erfordert. Hiervon wurde teilweise Gebrauch gemacht. So wurde etwa in Bayern die Meldepflicht auf die nichtnamentliche Meldung der Erkrankung und des Todes durch Borreliose in Form eines Erythema migrans,

9 §1 Verordnung über die Ausdehnung der Meldepflicht auf Infektionen mit dem erstmals im Dezember 2019 in Wuhan/Volksrepublik China aufgetretenen neuartigen Coronavirus (,2019nCoV") vom 31.01.2020, BAnz AT 31.01.2020 V1. Die Verordnung tritt am 1. Februar 2021 außer Kraft, sofern nicht mit Zustimmung des Bundesrates etwas anderes verordnet wird.

10 Verordnung zur Anpassung der Meldepflichten an die epidemische Lage, BGBI. I 2016, 515.

11 Art. 3 Gesetz für den Schutz vor Masern und zur Stärkung der Impfprävention vom 20.02.2020, BGBI.I2020, 148 . 
einer akuten Neuroborreliose und einer akuten Lyme-Arthritis erweitert. ${ }^{12}$

Zur Meldung sind gemäß $\$ 8$ IfSG zahlreiche Personengruppen verpflichtet. Hierzu gehören u. a. der feststellende Arzt, in Krankenhäusern, Einrichtungen für ambulantes Operieren, Vorsorge- oder Rehabilitationseinrichtungen, Tageskliniken und anderen näher bezeichneten Gesundheitseinrichtungen auch der leitende Arzt, in Krankenhäusern mit mehreren selbständigen Abteilungen der leitende Abteilungsarzt, in Einrichtungen ohne leitenden Arzt der behandelnde Arzt. In den Fällen des $\$ 6$ Abs. 1 Nr. 1, 2 und 5 und Abs. 3 IfSG sind auch die Angehörigen eines anderen Heil- oder Pflegeberufs, der für die Berufsausübung oder die Führung der Berufsbezeichnung eine staatlich geregelte Ausbildung oder Anerkennung erfordert (so etwa Pflegefachkräfte mit der Erlaubnis zum Führen einer Berufsbezeichnung nach dem Krankenpflegeoder Pflegeberufegesetz, Notfallsanitäter, Rettungsassistenten), zur Meldung verpflichtet, sofern kein Arzt hinzugezogen wurde.

Für Personen des Not- und Rettungsdiensts besteht die Meldepflicht nicht, wenn der Patient unverzüglich in eine ärztlich geleitete Einrichtung gebracht wurde ( $\$ 8$ Abs. 2 IfSG). Hier soll die Meldung durch die Ärzte der aufnehmenden Einrichtung erfolgen. ${ }^{13}$

Eine Meldepflicht besteht auch dann nicht, wenn dem Meldepflichtigen ein Nachweis vorliegt, dass die Meldung bereits erfolgte und andere als die bereits gemeldeten Angaben nicht erhoben wurden ( $\$ 8$ Abs. 3 Satz 1 IfSG). Eine Meldepflicht besteht ebenfalls nicht für Erkrankungen, bei denen der Verdacht bereits gemeldet wurde und andere als die bereits gemeldeten Angaben nicht erhoben wurden ( $\$ 8$ Abs. 3 Satz 2 IfSG). Hiervon abweichend ist in Bezug auf eine Infekti-

\footnotetext{
12 Verordnung zur Erweiterung der Meldepflicht auf andere übertragbare Krankheiten oder Krankheitserreger (Meldepflichtverordnung - MeldePfIV) vom 14.02.2018, GVBI. 2018, 69.

13 Gesetzesentwurf der Bundesregierung zur Neuordnung seuchenrechtlicher Vorschriften, BT-Drucks. 14/2530, 65.
}

on, die durch das erstmals im Dezember 2019 in Wuhan/Volksrepublik China aufgetretene neuartige Coronavirus (2019$\mathrm{nCoV}$ ) hervorgerufen wird, eine Erkrankung auch dann zu melden, wenn der Verdacht bereits gemeldet wurde. Ebenfalls ist zu melden, wenn sich der Verdacht einer Infektion nicht bestätigt $(\$ 1$ Abs. 1 Sätze 2 und 3 Meldepflicht-Verordnung 2019-nCoV).

Die im Rahmen der namentlichen Meldung zu übermittelnden Angaben ergeben sich aus $\$ 9$ IfSG. ${ }^{14}$ Hierzu gehören neben zahlreichen Angaben zur betroffenen Person (u. a. Name, Geschlecht, Geburtsdatum, Anschrift der Hauptwohnung oder des gewöhnlichen und derzeitigen Aufenthaltsorts, weitere Kontaktdaten, Diagnose bzw. Verdachtsdiagnose, Tag der Erkrankung und der Diagnose, wahrscheinliche Infektionsquelle) auch der Name, die Anschrift und weitere Kontaktdaten des Meldenden. Die Meldung muss unverzüglich erfolgen und spätestens innerhalb von $24 \mathrm{~h}$ nach erlangter Kenntnis dem zuständigen Gesundheitsamt vorliegen. Zuständig ist in der Regel das Gesundheitsamt, in dessen Bezirk sich die betroffene Person derzeit aufhält oder zuletzt aufhielt. Eine Meldung darf wegen einzelner fehlender Angaben nicht verzögert werden. Die Nachmeldung oder Korrektur von Angaben hat unverzüglich nach deren Vorliegen zu erfolgen. Das Gesundheitsamt ist befugt, von dem Meldenden Auskunft über Angaben zu verlangen, die die Meldung zu enthalten hat.

\section{Maßnahmen zur Verhütung übertragbarer Krankheiten}

Werden Tatsachen festgestellt, die zum Auftreten einer übertragbaren Krankheit führen können, oder ist anzunehmen, dass solche Tatsachen vorliegen, etwa bei in der Vergangenheit wiederholt aufgetretenen Hygienemängeln in einer Arztpraxis, ${ }^{15}$ obliegt es der zuständigen Behörde, die notwendigen Maßnahmen

\footnotetext{
${ }^{14}$ Die Anforderungen an die nichtnamentliche Meldung nach $\S 6$ Abs. 3 IfSG finden sich in $\S 10$ IfSG.

15 VG Würzburg, Beschl. v. 17.10.2016, Az. 6

S16/993, BeckRS 2016, 53734.
}

Urologe $2020 \cdot 59: 607-612$

https://doi.org/10.1007/s00120-020-01212-x

○) Springer Medizin Verlag $\mathrm{GmbH}$, ein Teil

von Springer Nature 2020

\section{P. M. Lissel}

\section{Infektionsschutzrecht nach Inkrafttreten des Masernschutzgesetzes}

\section{Zusammenfassung}

Am 1. März 2020 traten die durch das Gesetz für den Schutz vor Masern und zur Stärkung der Impfprävention (Masernschutzgesetz) eingeführten Änderungen des Infektionsschutzgesetzes in Kraft. Hintergrund der Änderungen sind die in den letzten Jahren deutlich gestiegenen Masernfallzahlen. Zum Schutz der öffentlichen Gesundheit wurden mit dem Masernschutzgesetz Regelungen implementiert, wonach Personen in bestimmten Einrichtungen entweder einen ausreichenden Impfschutz gegen Masern oder aber eine Immunität gegen Masern aufweisen müssen. Der Beitrag stellt die aktuelle Rechtslage bezogen auf Gesundheitseinrichtungen dar.

Schlüsselwörter

Öffentliche Gesundheit · Masern .

Impfschutz · Immunität · Gesundheitseinrichtungen

\section{The German Protection} Against Infection Act after entry into force of the German Measles Protection Act

\section{Abstract}

On 1 March 2020, the amendments to the German Protection Against Infection Act that were introduced by the act to protect against measles and strengthen vaccination prevention (Measles Protection Act) entered into force. The reason for the changes is that the number of individuals with measles has significantly increased in recent years. To protect public health, the Measles Protection Act has implemented regulations requiring that persons in certain institutions must either have adequate protection against measles or have immunity to measles. In this article the current legal situation with regard to health care facilities is presented.

\section{Keywords}

Public health - Measles - Vaccination .

Immunity $\cdot$ Health care facilities 
zur Abwendung von drohenden Gefahren zu treffen ( $\$ 16$ Abs. 1 IfSG). Die zuständigen Behörden werden durch das jeweilige Landesrecht bestimmt.

Die Beauftragten der zuständigen Behörde und des Gesundheitsamts sind zur Durchführung von Ermittlungen und zur Überwachung der angeordneten Maßnahmen berechtigt, Grundstücke, Räume, Anlagen und Einrichtungen sowie Verkehrsmittel zu betreten, und Bücher oder sonstige Unterlagen einzusehen und hieraus Abschriften, Ablichtungen oder Auszüge anzufertigen sowie sonstige Gegenstände zu untersuchen oder Proben zur Untersuchung zu fordern oder zu entnehmen. Personen, die über Tatsachen Auskunft geben können, die zum Auftreten einer übertragbaren Krankheit führen, sind verpflichtet, auf Verlangen die erforderlichen Auskünfte insbesondere über den Betrieb und den Betriebsablauf einschließlich dessen Kontrolle zu erteilen sowie Unterlagen einschließlich dem tatsächlichen Stand entsprechender technischer Pläne vorzulegen. Der Verpflichtete kann die Auskunft auf solche Fragen verweigern, deren Beantwortung ihn selbst oder einen Angehörigen der Gefahr strafrechtlicher Verfolgung oder eines Verfahrens nach dem Ordnungswidrigkeitengesetz aussetzen würde; Entsprechendes gilt für die Vorlage von Unterlagen ( $\$ 16$ Abs. 2 IfSG).

Wenn Gegenstände mit meldepflichtigen Krankheitserregern behaftet sind oder wenn das anzunehmen ist und dadurch eine Verbreitung der Krankheit zu befürchten ist, hat die zuständige Behörde die notwendigen $\mathrm{Ma}$ nnahmen zur Abwendung der hierdurch drohenden Gefahren zu treffen. Wenn andere Maßnahmen nicht ausreichen, kann auch die Vernichtung der Gegenstände angeordnet werden. Müssen diese entseucht (desinfiziert), von Gesundheitsschädlingen befreit oder vernichtet werden, kann ihre Benutzung und die Benutzung der Räume und Grundstücke, in denen oder auf denen sie sich befinden, untersagt werden, bis die Maßnahme durchgeführt ist ( $\$ 17$ IfSG).

$\$ 20$ IfSG sieht Schutzimpfungen und andere Maßnahmen der spezifischen Prophylaxe vor. Die Bundeszentrale für gesundheitliche Aufklärung (BZgA), die obersten Landesgesundheitsbehörden und die Gesundheitsämter informieren die Bevölkerung über die Bedeutung von Schutzimpfungen und andere Maßnahmen der spezifischen Prophylaxe übertragbarer Krankheiten $(\$ 20$ Abs. 1 IfSG). Die beim RKI eingerichtete Ständige Impfkommission (STIKO) gibt Empfehlungen zur Durchführung von Schutzimpfungen und zur Durchführung anderer Maßnahmen der spezifischen Prophylaxe übertragbarer Krankheiten und entwickelt Kriterien zur Abgrenzung einer üblichen Impfreaktion und einer über das übliche Ausmaß einer Impfreaktion hinausgehenden gesundheitlichen Schädigung ( $\$ 20$ Abs. 2 IfSG).

Das Bundesministerium für Gesundheit kann durch Rechtsverordnung anordnen, dass bedrohte Teile der Bevölkerung an Schutzimpfungen oder anderen Maßnahmen der spezifischen Prophylaxe teilzunehmen haben, wenn eine übertragbare Krankheit mit klinisch schweren Verlaufsformen auftritt und mit ihrer epidemischen Verbreitung zu rechnen ist. Solange das Bundesministerium hiervon keinen Gebrauch macht, sind die Landesregierungen zum Erlass einer entsprechenden Rechtsverordnung ermächtigt ( $\$ 20$ Abs. 6, 7 IfSG).

Bestimmten Personengruppen, die nach dem 31. Dezember 1970 geboren sind (für diesen Personenkreis empfiehlt die STIKO Masernschutzimpfungen), werden gemäß $₫ 20$ Abs. 8 bis 14 IfSG weitere Pflichten auferlegt. Von diesen durch das Masernschutzgesetz eingeführten Vorschriften sind u. a. Personen umfasst, die in näher bezeichneten Gesundheitseinrichtungen wie Krankenhäusern, Einrichtungen für ambulantes Operieren, Vorsorge- oder Rehabilitationseinrichtungen, Tageskliniken, Arztpraxen, ambulanten Pflegediensten und Rettungsdiensten tätig sind. Dies betrifft insbesondere medizinisches Personal, erfasst werden aber auch andere dort tätige Personen wie zum Beispiel Küchen- oder Reinigungspersonal, ebenso ehrenamtlich Tätige und Praktikanten. ${ }^{16}$

\footnotetext{
16 Gesetzesentwurf der Bundesregierung für den Schutz vor Masern und zur Stärkung der Impfprävention, BT-Drucks. 19/13452, 28.
}

Diese Personen müssen nach $₫ 20$ Abs. 8 IfSG entweder einen ausreichenden Impfschutz gegen Masern oder eine Immunität gegen Masern aufweisen. Ausgenommen sind Personen, die aufgrund einer medizinischen Kontraindikation nicht geimpft werden können.

Die Personen sind verpflichtet, der Leitung der Gesundheitseinrichtung eine entsprechende Impfdokumentation nach $\$ 22$ IfSG (Impfausweis, Impfbescheinigung) oder ein ärztliches Zeugnis vorzulegen, dass bei Ihnen ein ausreichender Impfschutz gegen Masern besteht bzw. dass bei ihnen eine Immunität gegen Masern vorliegt oder sie aufgrund einer medizinischen Kontraindikation nicht geimpft werden können. Ein ausreichender Impfschutz besteht, wenn ab der Vollendung des ersten Lebensjahrs mindestens eine Schutzimpfung und ab der Vollendung des zweiten Lebensjahrs mindestens zwei Schutzimpfungen gegen Masern bei der betroffenen Person durchgeführt wurden. Eine Immunität gegen Masern kann durch einen Arzt bestätigt werden, wenn diesem eine frühere Masernerkrankung der Person bekannt ist oder eine serologische Titerbestimmung einen ausreichenden Immunschutz ergeben hat. ${ }^{17}$ Statt der Nachweisführung durch eine Impfdokumentation oder ein ärztliches Zeugnis ist auch eine Bestätigung einer staatlichen Stelle oder der Leitung einer anderen Gesundheitseinrichtung, in der die betroffene Person vorher tätig war, darüber ausreichend, dass ein Nachweis bereits vorgelegen hat. Hierdurch sollen die Leitungen von Gesundheitseinrichtungen entlastet werden.

Personen, die in einer Gesundheitseinrichtung tätig werden sollen, haben den Nachweis vor Beginn ihrer Tätigkeit $\mathrm{zu}$ erbringen. Verfügen sie über keinen Nachweis oder legen diesen nicht vor, dürfen sie in der Einrichtung nicht tätig werden. Personen, die am 1. März 2020 bereits in der Einrichtung tätig waren, haben den Nachweis bis zum Ablauf des 31. Juli $2021 \mathrm{zu}$ erbringen ( $\$ 20$ Abs. 9, 10 IfSG).

\footnotetext{
17 Gesetzesentwurf der Bundesregierung für den Schutz vor Masern und zur Stärkung der Impfprävention, BT-Drucks. 19/13452, 29.
} 


\section{Infobox}

Richtlinien, Empfehlungen und Merkblätter

zur Prävention, Erkennung und Verhinderung

der Weiterverbreitung übertragbarer

Krankheiten sowie weiterführende

Informationen und Literaturhinweise

zu Infektionskrankheiten und zum

Infektionsschutz finden sich auf der Website

des RKI (http://www.rki.de). Aktuelle Daten

und Informationen zu Infektionskrankheiten

bieten das (wöchentlich erscheinende)

Epidemiologische Bulletin und das

Infektionsepidemiologische Jahrbuch des RKI.

Durch die Datenbank „SurvStat@RKI“ (http:// www.survstat.rki.de) ist eine webbasierte Abfrage der Meldedaten möglich. Weitere Informationen zum Masernschutzgesetz finden sich auf der diesbezüglichen Website des BZgA (http://www.masernschutz.de).

Auf Anforderung des Gesundheitsamts haben die in einer Gesundheitseinrichtung tätigen Personen den Nachweis auch dem Gesundheitsamt vorzulegen ( $\$ 20$ Abs. 12 IfSG). Wenn der Nachweis nicht innerhalb einer angemessenen Frist vorgelegt wird, kann das Gesundheitsamt die zur Vorlage des Nachweises verpflichtete Person zu einer Beratung laden und hat diese zu einer Vervollständigung des Impfschutzes gegen Masern aufzufordern. Legt die Person trotz der Anforderung keinen Nachweis innerhalb einer angemessenen Frist vor, kann das Gesundheitsamt der Person untersagen, Räume der Gesundheitseinrichtung zu betreten.

Nach $₫ 23$ Abs. 3 IfSG haben die Leiter von Krankenhäusern, Einrichtungen für ambulantes Operieren, Vorsorge- oder Rehabilitationseinrichtungen, Tageskliniken und anderen näher bezeichneten Gesundheitseinrichtungen - hierzu zählen seit Inkrafttreten des Masernschutzgesetzes auch Rettungsdienste - sicherzustellen, dass die nach dem Stand der medizinischen Wissenschaft erforderlichen Maßnahmen getroffen werden, um nosokomiale Infektionen $\mathrm{zu}$ verhüten und die Weiterverbreitung von Krankheitserregern, insbesondere solchen mit Resistenzen, zu vermeiden. Ferner müssen die Leiter sicherstellen, dass innerbetriebliche Verfahrensweisen zur Infektionshygiene in Hygieneplänen festgelegt sind. Die Gesundheitseinrichtungen unterliegen der infektions- hygienischen Überwachung durch das Gesundheitsamt ( $\$ 23$ Abs. 5 IfSG).

\section{Bekämpfung übertragbarer Krankheiten}

Ergibt sich oder ist anzunehmen, dass eine Person krank, krankheitsverdächtig, ansteckungsverdächtig oder Ausscheider ist oder dass ein Verstorbener krank, krankheitsverdächtig oder Ausscheider war, übernimmt das Gesundheitsamt die erforderlichen Ermittlungen über Art, Ursache, Ansteckungsquelle und Ausbreitung der Krankheit ( $\$ 25$ IfSG). ${ }^{18}$ Betroffene Personen können vorgeladen und verpflichtet werden, Untersuchungen und Entnahmen von Untersuchungsmaterial an sich vornehmen $\mathrm{zu}$ lassen, insbesondere die erforderlichen äußerlichen Untersuchungen, Röntgenuntersuchungen, Tuberkulintestungen, Blutentnahmen und Abstriche von Haut und Schleimhäuten durch die Beauftragten des Gesundheitsamts zu dulden sowie das erforderliche Untersuchungsmaterial auf Verlangen bereitzustellen.

Werden Kranke, Krankheitsverdächtige, Ansteckungsverdächtige oder Ausscheider festgestellt oder ergibt sich, dass ein Verstorbener krank, krankheitsverdächtig oder Ausscheider war, so trifft die zuständige Behörde die notwendigen Schutzmaßnahmen ( $\$ \$ 28$ ff. IfSG). ${ }^{19}$ Betroffene Personen können einer Beobachtung unterworfen werden $(\$ 29$ IfSG), unter strengen Voraussetzungen kann auch eine Absonderung in einem Krankenhaus oder einer sonstigen geeigneten Einrichtung angeordnet werden (Quarantäne), was im Weigerungsfalle auch zwangsweise durch Unterbringung in einem abgeschlossenen Krankenhaus oder einem abgeschlossenen Teil eines

\footnotetext{
18 Im Einzelnen vgl. Lissel, Infektionsschutzrecht, in: Rieger et al., Heidelberger Kommentar Arztrecht Krankenhausrecht Medizinrecht, 72. Aktualisierung 2018, Ziffer 2605 RN $88 \mathrm{ff}$.

19 Hierauf stützen sich jüngst zahlreiche Verordnungen und Verfügungen im Zusammenhang mit dem neuartigen, erstmals im Dezember 2019 in Wuhan/Volksrepublik China aufgetretenen Coronavirus SARS-CoV-2 (2019-nCoV) und die durch das Virus ausgelöste Atemwegserkrankung COVID-19.
}

Krankenhauses durchgesetzt werden kann ( $\$ 30$ IfSG).

\section{Bußgeld- und Strafvorschriften}

$\$ 73$ IfSG enthält zahlreiche Bußgeldvorschriften. So handelt u.a. ordnungswidrig, wer entgegen $\$ 6$ Abs. 1 IfSG eine Meldung nicht, nicht richtig, nicht vollständig, nicht in der vorgeschriebenen Weise oder nicht rechtzeitig macht. Ordnungswidrig handelt ferner, wer entgegen $₫ 16$ Abs. 2 IfSG ein Grundstück, einen Raum, eine Anlage, eine Einrichtung, ein Verkehrsmittel oder einen sonstigen Gegenstand nicht zugänglich macht oder eine Auskunft oder Unterlage nicht, nicht richtig, nicht vollständig oder nicht rechtzeitig erteilt bzw. vorlegt. Auch handelt ordnungswidrig, wer entgegen $\$ 20$ Abs. 9 IfSG in einer Gesundheitseinrichtung tätig wird, ohne über einen entsprechenden Nachweis (Impfdokumentation oder ärztliches Zeugnis bzw. Bestätigung einer staatlichen Stelle oder einer anderen Gesundheitseinrichtung) zu verfügen oder diesen vorzulegen. Ordnungswidrig handelt auch, wer einen entsprechenden Nachweis entgegen $\$ 20$ Abs. 12 IfSG dem Gesundheitsamt nicht, nicht richtig, nicht vollständig oder nicht rechtzeitig vorlegt oder einem Betretungsverbot des Gesundheitsamts zuwiderhandelt. Die Ordnungswidrigkeiten können mit einer Geldbuße bis zu $€ 25.000$ geahndet werden. Landesrechtliche Vorschriften, die die Meldepflichten des IfSG für das jeweilige Bundesland ergänzen, enthalten teilweise ebenfalls Bußgeldvorschriften.

Teilweise werden die Ordnungswidrigkeiten auch zu Straftaten, wenn sie vorsätzlich begangen werden und sich dadurch eine in $\$ 6$ Abs. 1 Satz 1 Nr. 1 IfSG genannte Krankheit verbreitet. Der Strafrahmen reicht von einer Geldstrafe bis $\mathrm{zu}$ einer Freiheitsstrafe bis $\mathrm{zu}$ fünf Jahren ( $\$ 74$ IfSG).

Nach $₫ 75$ IfSG macht sich strafbar, wer bestimmten Schutzmaßnahmen zur Bekämpfung übertragbarer Krankheiten zuwiderhandelt. Der Strafrahmen reicht hier von einer Geldstrafe bis $\mathrm{zu}$ einer Freiheitsstrafe bis zu fünf Jahren. 


\section{Klinik- und Praxismanagement}

\section{Korrespondenzadresse}

\section{Dr. P. M. Lisse}

München, Deutschland

Dr. P.M. Lissel LL.M. Rechtsanwalt und Fachanwalt für Medizinrecht

\section{Einhaltung ethischer Richtlinien}

Interessenkonflikt. P.M. Lissel gibt an, dass kein Interessenkonflikt besteht.

Für diesen Beitrag wurden vom Autor keine Studien an Menschen oder Tieren durchgeführt. Für die aufgeführten Studien gelten die jeweils dort angegebenen ethischen Richtlinien.

\section{Praxis-Finanzrechner für Ärzte Neues Tool auf SpringerMedizin.de}

Wie rentabel läuft meine Praxis? Wie stehe ich im Vergleich zu meinen Kollegen im Bundesland? Welche Anschaffungen kann ich mir leisten? Antworten auf diese betriebswirtschaftlichen Fragen liefert künftig ein Tool auf SpringerMedizin.de, indem es aktuelle Versorgungs- und Abrechnungsdaten sowie Analysedaten deutscher Gesundheitsunternehmen zu Grunde legt.

\section{Mit den kostenfreien Praxis-Finanzrechnern können sich vor} allem in der Praxis tätigen Ärzte, die sich oft auch unternehmerischen Herausforderungen stellen müssen, interaktiv mit den Themen Controlling und Benchmarking auseinandersetzen:

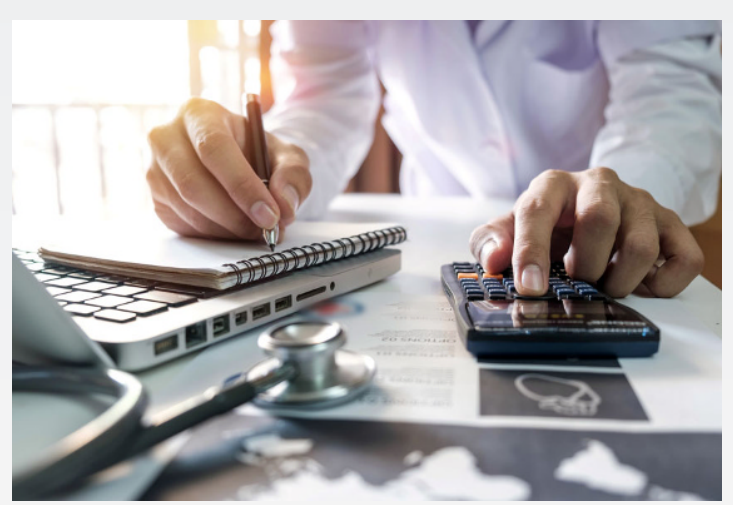

(c) mrmohock/stock.adobe.com
Mit dem Controlling-Assistenten behalten Sie die wichtigsten wirtschaftlichen Praxiskennzahlen immer im Auge. $>$ Mit dem Tool Benchmark können Sie Ihre Praxis mit anderen Praxen vergleichen.

> Ihren Praxisstandort - ebenfalls ein wichtiger Faktor für den Erfolg - analysieren Sie mit Standortcheck aus verschiedenen Perspektiven.

> Mit dem Investitionsassistenten können sie sehen, ob und wann sich eine Geräteinvestition amortisiert und Sie bekommen auch Anhaltspunkte, welche Einnahmen Sie zu erwarten haben.

Die Praxis-Tools sind ein externes Angebot von Rebmann Research, Partner von SpringerMedizin.de. Sie finden die Praxis-Finanzrechner auf SpringerMedizin.de unter dem Navigationspunkt "Mehr".

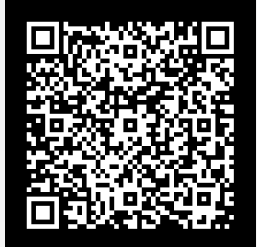

